\title{
FILMES A BASE DO SUBPRODUTO DA EXTRAÇÃO DO ÓLEO DE BABAÇU PLASTIFICADOS COM GLICEROL E SORBITOL
}

\author{
B. C. Maniglia ${ }^{1}$, A. de A. Lucas $^{2}$, D. R. Tapia-Blácido ${ }^{1}$ \\ ${ }^{1}$ Universidade de São Paulo, Departamento de Química, Faculdade de Filosofia, Ciências e \\ Letras de Ribeirão Preto. \\ ${ }^{2}$ Universidade de São Carlos, Departamento de Engenharia de Materiais. \\ E-mail para contato: biancamaniglia@gmail.com
}

RESUMO - O interesse industrial do babaçu está na extração de óleo das amêndoas, sendo as demais partes do fruto, descartadas. $O$ resíduo, principalmente, o mesocarpo de babaçu, apresenta um importante teor de amido sendo interessante para a produção de filmes biodegradáveis. O objetivo deste trabalho foi avaliar a capacidade filmogênica do material extraído a partir do mesocarpo de babaçu. Também foi avaliado o efeito do glicerol e sorbitol nas propriedades mecânicas, solubilidade, molhabilidade, cristalinidade, barreira ao vapor de água e microestrutura dos filmes produzidos. A composição centesimal, a cristalinidade por DRX e a microestrutura do material extraído também foram determinados. Os filmes foram produzidos por casting a partir de uma suspensão de $4 \%(\mathrm{p} / \mathrm{p})$ utilizando glicerol e sorbitol como plastificante (19 g plastificante/100 g de farelo). O material extraído por moagem úmida do mesocarpo de babaçu apresentou alto teor de amido e baixo teor de cinzas, proteínas, lipídeos e fibras. O filme preparado com glicerol apresentou maior resistência mecânica, maior elongação, maior solubilidade, menor barreira ao vapor de água e microestrutura mais regular do que filmes plastificados com sorbitol. Portanto, o glicerol foi o plastificante mais compatível com esta matriz polimérica do que o sorbitol.

\section{INTRODUÇÃO}

O crescente acúmulo de lixo não biodegradável, aliado à dificuldade de reciclagem da maioria das embalagens sintéticas na atualidade, tem lançado um desafio à comunidade científica mundial no sentido de desenvolver novos materiais biodegradáveis. Diante desse contexto de busca por soluções que minimizem a poluição, estudos estão sendo elaborados com o objetivo de formar filmes biodegradáveis a partir de resíduos ou subprodutos gerados pelas indústrias. Este é o caso do mesocarpo de babaçu que é um resíduo proveniente da extração do óleo das amêndoas do coco de babaçu.

O babaçu (Orbignya phalerata Mart.) é uma palmeira que nasce naturalmente no norte do Brasil, nos estados de Piauí, Tocantins e Maranhão, também no Cerrado e na floresta Amazônica, em um ecosistema chamado "Mata dos Cocais". O fruto é uma drupa com elevado número de frutos por cacho, sendo estes em número de 4 (habitat natural), que varia de 15 a 25. Os frutos são em formato elipsoidal, mais ou menos cilíndricos, pesando de 90 a 
280 g. Este fruto apresenta: epicarpo (camada mais externa e bastante rija), mesocarpo (com 0,5 a 1,0 cm e rico em amido), endocarpo (rijo, de 2 a $3 \mathrm{~cm}$ ) e amêndoas (de 3 a 4 por fruto, com 2,5 a $6 \mathrm{~cm}$ de comprimento e 1 a $2 \mathrm{~cm}$ de largura). Os pesos médios de cada componente do coco babaçu são $11 \%$ de exocarpo, $23 \%$ de mesocarpo, $59 \%$ de endocarpo - camada de madeira dura - e kernels 7\% (Teixeira, 2008).

Os principais produtos comerciais extraídos do Babaçu são o óleo (extraído da castanha) e a torta (porque resulta do processo de extração do óleo). Este óleo representa 7\% do peso total do fruto, sendo o restante um resíduo. O endocarpo (parte lenhosa) possui elevado conteúdo de carbono fixo, sendo o mais indicado tanto para a carbonização quanto para a queima e o epicarpo (casca), por sua vez, mostra-se um bom combustível, porém apresenta baixo potencial de carbonização. E finalmente, o mesocarpo com menos de $5 \%$ de carbono fixo, mostra-se inadequado tanto para carbonização como para a queima. Portanto, o mesocarpo não é um bom material para ser utilizado como biomassa, sendo necessário encontrar outra utilidade para tal resíduo (Teixeira, 2008).

O óleo de babaçu apresenta aplicações industriais como em alimentos, detergentes e cosméticos e também para produção de biodiesel (De Medeiros et al., 2013). Em 2011, 102.499 toneladas de coco babaçu foram colhidas no Brasil, principalmente na região Nordeste região (IBGE, 2011).

O mesocarpo do fruto de babaçu apresenta ao redor de $63,75 \%$ a $71,29 \%$ de amido (Miotto et al., 2013). Os grânulos de amido de babaçu têm uma estrutura similar à dos cereais, mas diferente de raízes e tubérculos como, por exemplo, a mandioca e a batata. A temperatura de gelatinização é em torno de $63^{\circ} \mathrm{C}$ e $73^{\circ} \mathrm{C}$ devido ao teor significativo de amilose. Devido à composição rica em amido deste resíduo, este material se apresenta como um material promissor na produção de filmes biodegradáveis.

Este trabalho teve como objetivo avaliar o potencial da farinha obtida a partir do mesocarpo do coco babaçu na formação de filmes através da verificação de suas características mecânicas e funcionais. Também foi avaliada a interação dessa matriz com dois tipos diferentes de plastificantes: glicerol e sorbitol.

\section{MATERIAIS E MÉTODOS}

O mesocarpo de coco Babaçu foi fornecido pela Coopaesp, empresa cooperativa presente em Esperantinópolis - Maranhão. O material recebido passou por moagem úmida seguida de peneiragem (80, 200 e 270 mesh). O material retido nas peneiras foi seco, moído e peneirado em peneira de aço inox de 400 mesh de abertura. Este material foi chamado de farinha de babaçu.

\subsection{Caracterização da farinha de babaçu}

Os teores de umidade, proteínas e cinzas foram determinados de acordo com o método oficial da AOAC (1997), os lipídeos pelo método de Bligh-Dyer (Blig \& Dier, 1959) e o teor de fibras de acordo com o método descrito na norma TAPPI T19 m-54. O teor de amido foi calculado por diferença. A microestrutura da farinha de babaçu foi analisada por microscopia 
eletrônica de varredura da marca ZEISS modelo EVO-50, a $20 \mathrm{kV}$. A cristalinidade foi determinada através de difração de raios-X em um espectrofotômetro de raios X Philips 1730 , entre $2 \theta=5$ a $2 \theta=40^{\circ}$ e velocidade $1^{\circ} 2 \theta / \mathrm{min}$.

\subsection{Produção dos filmes}

Os filmes foram elaborados por casting. Inicialmente foi preparada uma suspensão de $4 \%$ (p/p) em água deionizada, e homogeneizada por 30 minutos utilizando um agitador magnético (IKA® C-MAG HS7-Marconi). Despois a suspensão foi aquecida a $81^{\circ} \mathrm{C}$ por 30 minutos e adicionado o plastificante ( $19 \mathrm{~g}$ glicerol ou sorbitol/ $100 \mathrm{~g}$ de farinha). A suspensão foi aquecida por mais 15 minutos. Posteriormente, a solução foi adicionado nas placas de acrílico ou teflon mantendo a gramatura de $0,15 \mathrm{~g} / \mathrm{m}^{2}$ e foi iniciado o período de secagem durante $12 \mathrm{~h}$ em estufa com circulação forçada (Q314M, QUIMIS $\circledR$ ) a $35^{\circ} \mathrm{C}$. Os filmes secos foram cortados em moldes e acondicionados em atmosfera a 54\% UR (solução saturada de $\mathrm{NaBr})$.

\subsection{Caracterização dos filmes}

Propriedades mecânicas: $\mathrm{O}$ teste mecânico de tração dos filmes foi realizado em um Texturômetro TA. TX Plus (TA Instrument). A tensão e a elongação na ruptura em teste de tração foram determinadas segundo a norma ASTM D 882-95 (ASTM, 1995). A tensão (T) e elongação (E) na ruptura foram obtidas da curva de tensão versus elongação e o módulo de Young (MY) foi obtido como a inclinação da curva no intervalo linear.

Umidade, Solubilidade e permeabilidade ao vapor de água: A umidade dos filmes foi determinada segundo a técnica da ASTM D644-94 (ASTM, 1994). A solubilidade foi medida segundo a metodologia proposta por Gontard et al. (1992). A permeabilidade ao vapor de água foi determinada segundo o método padrão modificada ASTME E96-95 (ASTM, 1995).

Cristalinidade dos filmes: A cristalinidade dos filmes foi determinada através de um espectrofotômetro de raios X Philips 1730 , entre $2 \theta=5^{\circ}$ a $2 \theta=40^{\circ}$ e velocidade $1^{\circ} 2 \theta / \mathrm{min}$. A partir dos difratogramas de raios $\mathrm{X}$ dos filmes foram calculados os índices de cristalinidade, que representam a razão entre a área do pico de difração e a área total de difração.

Análises Térmicas dos filmes por Calorimetria Diferencial de Varredura (DSC): As transições de fase dos filmes plastificados com glicerol e com sorbitol foram determinadas por análise calorimétrica de varredura, utilizando um DSC TA 2010, controlado por um módulo TA5000 (TA Instruments, New Castle, DE, EUA) acoplado a um acessório de refrigeração crioscópica. As amostras foram aquecidas a uma taxa de $10^{\circ} \mathrm{C} / \mathrm{min}$, entre -150 a $150^{\circ} \mathrm{C}$, em ambiente inerte $\left(45 \mathrm{~mL} / \mathrm{min}\right.$ de $\left.\mathrm{N}_{2}\right)$. Foi utilizado, para referência, uma cápsula vazia, e o equipamento foi calibrado com amostra de índium $\left(\mathrm{Tm}=156,6^{\circ} \mathrm{C}\right.$ e $\left.\mathrm{Hm}=28,71 \mathrm{~J} / \mathrm{g}\right)$ (TA Instruments). Os resultados obtidos foram analisados com o auxílio do software Universal Analyser TA Software.

Ângulo de contato dos filmes: As medidas de ângulo de contato foram obtidas empregando-se o OCA-20-Dataphysiscs, tomando-se imagens de uma gota de água (tensão superficial $72,7 \mathrm{mN} / \mathrm{m}$ ) por um intervalo de dois minutos operado em ar e a temperatura ambiente. $\mathrm{O}$ medidor de ângulo de contato utiliza uma câmera para aquisição das imagens e 
uma fonte de luz para a formação de uma imagem nítida da gota do líquido sobre a superfície estudada. Foi utilizado o software de tratamento de imagem GIMP 2.6.8 para aferição do ângulo de contato, que é identificado na tangente à borda da gota, partindo a intersecção dos meios: líquido, sólido e gasoso.

Microestrutura dos filmes: Amostras de filmes foram fraturadas em $\mathrm{N}_{2}$ líquido para analisar a microestrutura superficial e a seção transversal. Depois foram recobertos com ouro e analisados em um microscópio eletrônico de varredura da marca ZEISS modelo EVO-50, a $20 \mathrm{kV}$.

\subsection{Análise estatística}

Para avaliar as diferenças nas propriedades dos filmes plastificados com glicerol e sorbitol foi realizado o teste de Tukey a 95\% de nível de significância $(\mathrm{p}<0.05)$ utilizando o software Statistica 6.0.

\section{RESULTADOS E DISCUSSÕES}

\subsection{Caracterização da farinha de babaçu}

A farinha de babaçu apresentou baixo conteúdo de cinzas $(0,96 \% \pm 0,23$ b.s $)$, lipídeos $(0,19 \% \pm 0,07$ b.s $)$, proteínas $(2,65 \% \pm 0,07$ b.s $)$, fibras $(3,30 \% \pm 0,22$ b.s $)$ e um alto teor de amido (93\%). A umidade foi de 9,58\% $\pm 0,89$ (base úmida).

$\mathrm{Na}$ Figura 1 mostra-se a microestrutura da farinha de babaçu obtida por Microscopia Eletrônica de Varredura.

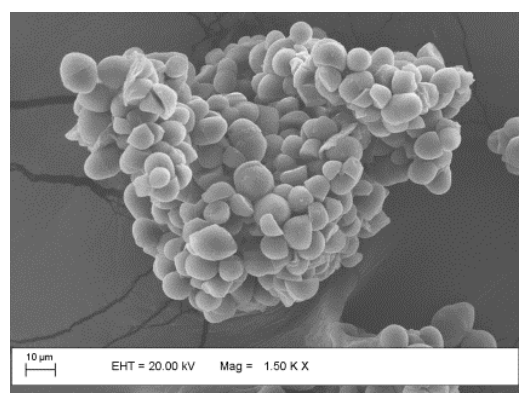

Figura 1 - Microestrutura da farinha de babaçu (aumento de 1500x)

A micrografia da farinha de babaçu revela a presença de grânulos de amido de formato poligonal ovalado, sem danos estruturais e de diferentes tamanhos $(\sim 10 \mu \mathrm{m})$. Também se observa que estes grânulos de amido encontram-se formando aglomerados, possivelmente devido à presença das proteínas, lipídeos e fibras.

A partir da análise de difração de raios-X da farinha de babaçu (Figura 2) nota-se a presença de cinco picos, um mais acentuado por volta de $17^{\circ}$ e os picos menores por volta de $5^{\circ}, 15^{\circ}, 22^{\circ}$ e $24^{\circ}$, indicando que o amido presente neste material é do tipo-B. O amido de babaçu se assemelha ao amido de inhame (Hoover, 2001) e ao amido de C. edulis por possuírem padrão de difração de raios-X tipo-B. 


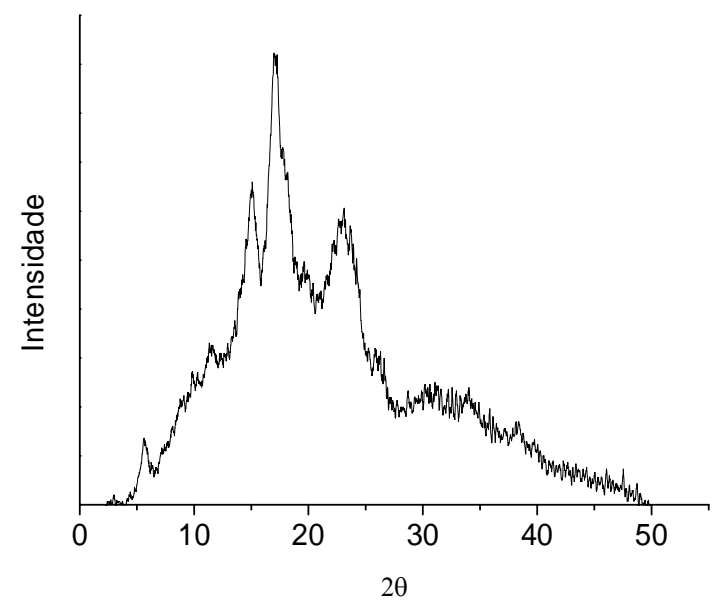

Figura 2 - Difratograma de raios -X da farinha de babaçu.

\subsection{Caracterização dos filmes de farinha de babaçu}

A Tabela 1 apresenta as propriedades mecânicas e funcionais dos filmes de farinha de babaçu plastificados com glicerol e sorbitol.

Tabela 1 - Propriedades dos filmes de babaçu plastificados com glicerol e sorbitol

\begin{tabular}{|c|c|c|}
\hline Propriedades & Glicerol & Sorbitol \\
\hline Tensão (MPa) & $43,6 \pm 7,5^{\mathrm{a}}$ & $15,4 \pm 2,2^{b}$ \\
\hline Elongação (\%) & $4,0 \pm 0,4^{\mathrm{a}}$ & $1,0 \pm 0,3^{\mathrm{b}}$ \\
\hline Módulo de Young (MPa) & $2372,3 \pm 465^{\mathrm{a}}$ & $1474,8 \pm 513^{b}$ \\
\hline PVAx $10^{-10}\left(\mathrm{~g} \cdot \mathrm{m}^{-1} \cdot \mathrm{s}^{-1} \cdot \mathrm{Pa}^{-1}\right)$ & $11,6 \pm 0,1^{\mathrm{a}}$ & $3,1 \pm 0,5^{\mathrm{b}}$ \\
\hline Solubilidade (\%) & $27,5 \pm 0,5^{\mathrm{a}}$ & $13,3 \pm 0,8^{b}$ \\
\hline Umidade $(\%)$ & $12,2 \pm 0,7^{\mathrm{a}}$ & $9,7 \pm 0,7^{b}$ \\
\hline Ângulo de contato & 75,0 & 67,5 \\
\hline Espessura (mm) & $0,088 \pm 0,003^{b}$ & $0,094 \pm 0,005^{\mathrm{a}}$ \\
\hline
\end{tabular}

a, b: letras minúsculas diferentes representa diferença significativa entre os plastificante segundo o teste de Tukey, $\mathrm{p}<0,05$.

O filme de farinha de babaçu plastificado com glicerol apresentou maior tensão, elongação e módulo de Young do que o filme plastificado com sorbitol. As diferenças entre estes plastificantes podem estar relacionadas com os seus pesos moleculares (PM). Plastificantes com menor PM, como o glicerol ( $92 \mathrm{~mol} / \mathrm{g})$, exercem plastificação mais efetiva que os de maior PM, como o sorbitol (182 mol/g) (Tapia-Blácido et al., 2011).

O filme plastificado com sorbitol apresentou menor valor de permeabilidade ao vapor de água, menor solubilidade e menor umidade que o filme plastificado com glicerol. $\mathrm{O}$ 
glicerol e sorbitol são polióis, no entanto, o glicerol possui 3 carbonos ligados a 3 hidroxilas, e o sorbitol, 6 carbonos ligados a 6 hidroxilas, o que faz com que tenha uma maior capacidade de interação com as moléculas de amido através dos grupos hidroxilas da amilose e amilopectina. Desta forma, diminuem os grupos hidroxilas disponíveis para a interação com as moléculas de água. Portanto em presença do sorbitol o filme de babaçu é menos solúvel e menos permeável ao vapor de água. Além disso, o glicerol é mais hidrofílico do que o sorbitol, A higroscopicidade do sorbitol é baixa devido a sua capacidade de cristalizar a temperatura ambiente e alta umidade relativa (Talja et al., 2007).

O ângulo de contato dos filmes de farinha de babaçu plastificados com glicerol e sorbitol foram menores de $90^{\circ}$, o qual indica que ambos os filmes apresentam característica hidrofílica (Bico et. al, 1999).

A análise térmica dos filmes permitiu obter a temperatura de transição de fase ( $\mathrm{Tg})$ dos filmes de farinha de babaçu (Tabela 2). Observa-se que ambos os filmes apresentaram duas Tgs. $\mathrm{O}$ valor de $\mathrm{T}_{\mathrm{g} 1}$ foi menor que $0^{\circ} \mathrm{C}$ e está relacionada com a fase rica de plastificante, sendo este valor maior para o sorbitol. A segunda temperatura de transição vítrea $\left(\mathrm{T}_{\mathrm{g} 2}\right)$ está presente em ambos os filmes e corresponde à fase rica em amido. Pode-se notar que o valor de $T_{g_{2}}$ do filme plastificado com sorbitol é maior do que o plastificado com glicerol. Estes valores indicam que em temperatura ambiente $\left(\mathrm{T}<40^{\circ} \mathrm{C}\right)$, os filmes apresentam estabilidade térmica, maior resistência mecânica e menos flexibilidade.

Tabela 2 - Temperatura de transição de fase (Tg) dos filmes de farinha de babaçu plastificados com glicerol e sorbitol

\begin{tabular}{ccc}
\hline Plastificante & $\operatorname{Tg}_{1}\left({ }^{\circ} \mathrm{C}\right)$ & $\operatorname{Tg}_{2}\left({ }^{\circ} \mathrm{C}\right)$ \\
\hline Glicerol & $-68,82$ & 40,14 \\
Sorbitol & $-21,72$ & 49,63 \\
\hline
\end{tabular}

$\mathrm{Na}$ Figura 3 apresentam-se os difratogramas dos filmes de farinha de babaçu plastificados com glicerol e sorbitol. Nestes difratogramas foram observados picos em $5^{\circ}, 17^{\circ}$ e $20^{\circ}$ para ambos os filmes. O pico em $12^{\circ}$ foi mais evidente nos filmes de farinha de babaçu. Estes valores indicam que houve um deslocamento dos picos nos filmes de farinha de babaçu quando comparado com a matéria prima (Figura 2) devido à presença de água, glicerol e sorbitol nos filmes. A partir destes difratogramas de raios-X foi possível obter o índice de cristalinidade de cada filme. Os filmes de farinha de babaçu plastificados com glicerol apresentaram maior índice de cristalinidade $(37,98 \%)$ do que os filmes de sorbitol $(27,71 \%)$. Devido a isto, os filmes com glicerol apresentaram maior resistência mecânica à ruptura do que os filmes com sorbitol. A maior cristalinidade também indica um maior ordenamento molecular e menores espaços livres ou volume livre entre as moléculas. Portanto, pode-se dizer que em presença do glicerol, a matriz formada pelo amido e proteína ficou mais ordenada e compactada. O glicerol foi bem incorporado na matriz, interagindo com os polímeros presentes (amido e proteína), contribuindo a aumentar a força de interação entre as estruturas e promovendo um maior ordenamento. 


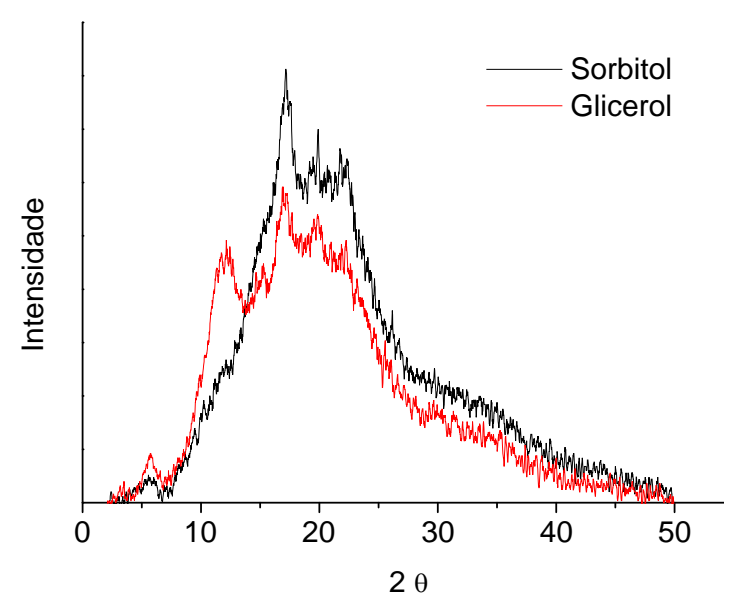

Figura 3 - Difratograma de raios $-X$ dos filmes de farinha de babaçu plastificados com glicerol e com sorbitol

Na Figura 4 estão apresentadas as micrografias dos filmes plastificados com glicerol e com sorbitol. Observa uma aparência mais rugosa para os filmes elaborados com o sorbitol do que com glicerol. A estrutura mais compactada no filme com glicerol proporcionou maior tensão na ruptura do que para os filmes com sorbitol.

(1)

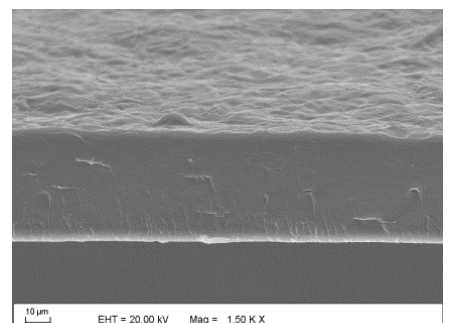

(2)

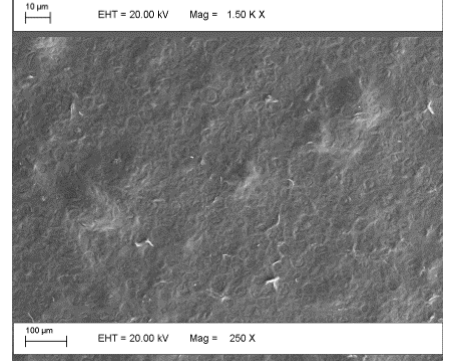

Glicerol
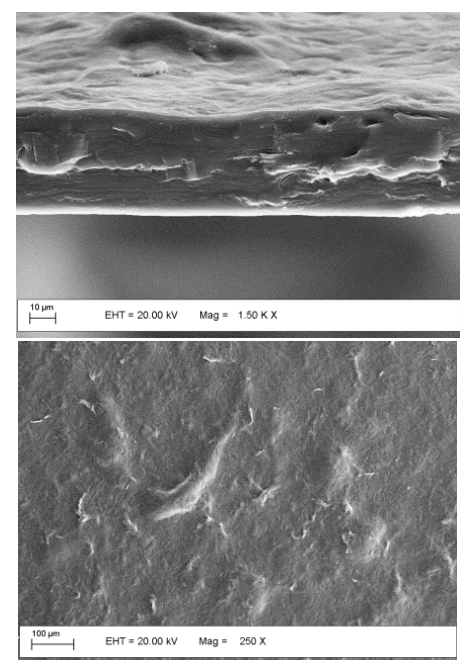

Sorbitol

Figura 4 - Microscopia da (1) vista transversal e (2) horizontal dos filmes produzidos a partir da farinha de babaçu plastificados com glicerol e sorbitol

\section{CONCLUSÃO}

A farinha de babaçu obtido a partir do resíduo do mesocarpo se apresentou como material promissor para a formulação de filmes, sendo este material mais barato por ser extraído a partir de um resíduo. O filme preparado com glicerol foi mecanicamente mais resistente, mais flexível, mais solúvel e mais permeável ao vapor de água. A microestrutura 
dos filmes de farinha de babaçu plastificados com glicerol foi mais densa e regular do que filmes plastificados com sorbitol, demonstrando maior compatibilidade com a matriz do filme de babaçu.

\section{AGRADECIMENTOS} CAPES.

Os autores agradecem o financiamento da FAPESP (Processo $N^{\circ}$ 2009/1460-0) e à

\section{REFERÊNCIAS BIBLIOGRÁFICAS}

AOAC. $16^{\text {a }}$ Edition. Washington: Association of Official Analytical Chemists, 1997.

ASTM. Standard test method for tensile properties of thin plastic sheeting. In: Annual book of American Standard Testing Methods. D882-95. Philadelphia, Pa.: ASTM, 1995.

ASTM. Standard test method of water vapor transmission of materials. In: Annual book of American Standard Testing Methods. E96-95. Philadelphia, Pa.: ASTM,1995.

ASTM. Standard test methods for moisture content of paper and paperboard by oven drying. Method D644-94. In Annual Book of ASTM Standards, American Society for Testing and Materials: Philadelphia, 1994.

BLIGH, E. G.; Dyer, W. J. A rapid method of total lipid extraction and purification. Can J. Biochem Physiol., v.37, p. 911-917, 1959.

BICO, J.; MARZOLIN, C.; QUERE, D. Pearl drops. Europhys. Lett., v. 47, n 2, p. $220,1999$.

DE MEDEIROS, E.J.L.; QUEIROGA, R.C.; DE SOUZA, A.G.; CORDEIRO, A.M.; DE MEDEIROS, A.N.; DE SOUZA, D.L.; MADRUGA, M.S. Thermal and quality evaluation of vegetable oils used in ruminant feed. J Therm Anal Calorim, v.112, p. 1515-1521, 2013.

GONTARD, N.; GUILBERT, S.; CUQ, J. L. Edible wheat gluten films: influence of the main process variables on film properties using response surface methodology. J. Food Sci., v. 57, n. 1, p. 190-195, 1992.

HOOVER, R. Composition, molecular structure, and physicochemical properties of tuber and root starches: A Review. Carbohyd. Polym., v. 45, n. 3, p. 253-267, 2001.

IBGE. Quantidade produzida na extração vegetal por tipo de produto extrativo. Sistema IBGE de recuperação automática - SIDRA: IBGE - Produção da Extração Vegetal e da Silvicultura; 2011.

MIOTTO, F.R.C.; RESTLE, J.; NEIVA, J.N.; CASTRO, N.; SOUSA, L.F.; DA SILVA, R., DE FREITAS, B., LEÃO, J. Replacement of corn by babassu mesocarp bran in diets for feedlot young Bulls. Rev. Bras. Zootecn., v.42, n.3, p. 213-219, 2013.

TAPIA-BLÁCIDO, D.; SOBRAL, P. J. A.; MENEGALLI, F. C. Optimization of amaranth flour films plasticized with glycerol and sorbitol by multi-response analysis. Food Sci Technol-LEB, v.44, n.8, p.1731-1738, 2011.

TALJA, R. A.; HELÉN, H.; ROOS, Y. H.; JOUPPILA, K. Effect of various polyols and polyol contents on physical properties of potato starch-based films. Carbohyd. Polym., v.67, n.3, p.288-295, 2007.

TAPPI STANDARD. -“Method T19 o-54”, TAPPI Test Methods, 1991.

TEIXEIRA, M. A. Babassu - A new approach for an ancient Brazilian biomass. Biomass Bioenerg., v. 32, p.857-864, 2008. 